\title{
Chapter 28 \\ The Computer-Controlled \\ Multicompartmental Dynamic Model of the Gastrointestinal System SIMGI
}

\author{
Elvira Barroso, Carolina Cueva, Carmen Peláez, M. Carmen \\ Martínez-Cuesta, and Teresa Requena
}

\begin{abstract}
The SIMGI (SIMulator Gastro-Intestinal) is an automated gastrointestinal in vitro model designed to dynamically simulate the physiological processes taking place during digestion in the stomach and small intestine, as well as to reproduce the colonic microbiota responsible for metabolic bioconversions in the large intestine. This computer-controlled system is a flexible modulating system that combines a gastric compartment that operates with peristaltic mixing movements, a reactor simulating the small intestine and three-stage continuous reactors that reproduce the colon region-specific microbiota. The compartments designed for digestion (stomach and small intestine) and fermentation (colon) can be connected to operate jointly. Alternatively, the digestion and fermentation processes can proceed independently. This section describes the conditions needed to inoculate, stabilize and differentiate the fecal microbiota in the SIMGI system, as well as the steps to follow in order to test the stabilized colonic microbiota with different food ingredients and/or by modifying the caloric intake in the nutrition media.
\end{abstract}

Keywords Automated in vitro dynamic model $\bullet$ Three-stage fermentation $\bullet$ SIMGI

- Colon microbiota

E. Barroso • C. Cueva • C. Peláez • M.C. Martínez-Cuesta • T. Requena ( $₫)$

Department of Food Biotechnology and Microbiology, Institute of Food Science Research,

CIAL (CSIC-UAM), Madrid, Spain

e-mail: t.requena@csic.es 


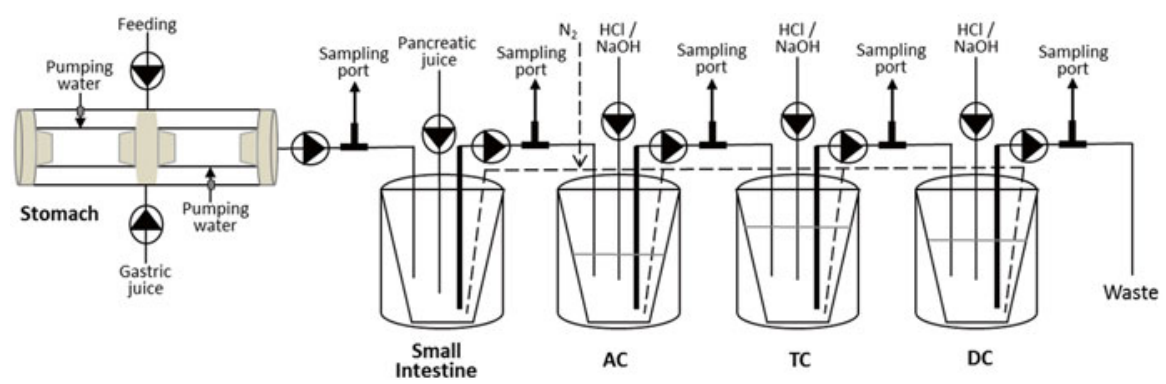

Fig. 28.1 Schematic diagram of the SIMulator Gastro-Intestinal SIMGI

\subsection{Description of the Model}

\subsubsection{History and Special Features of the Model}

The SIMGI model is a fully automated gastrointestinal multichamber simulator that has been recently developed at the Institute of Food Science Research CIAL (CSICUAM, Madrid, Spain). The SIMGI comprises five interconnected compartments (units) that simulate the stomach, small intestine and three stages of the large intestine (Fig. 28.1). The process of digestion is simulated in units Stomach and Small Intestine. Unit Stomach is comprised of two cylindrical transparent and rigid methacrylate plastic modules covering a reservoir of flexible silicone walls where the gastric content is mixed by peristaltic movements. The simulation of gastric peristalsis is achieved by changing the pressure of the water that flows in the jacket between the plastic modules and the reservoir. The pumped thermostated water keeps the temperature of the gastric content at $37^{\circ} \mathrm{C}$. The stomach compartment has different ports for input of experimental food components, gastric juice, and $\mathrm{HCl}$. The $\mathrm{pH}$ decrease is computer-controlled to follow the curve resulting from a linear fit of data representing experimental in vivo conditions. The small intestine consists in a double jacket glass reactor vessel continuously stirred that receives the gastric content which is mixed with pancreatic juice and bile. The intestinal content is digested during $2 \mathrm{~h}$ at $37^{\circ} \mathrm{C}$ and kept at $\mathrm{pH}$ 6.8. The stages of the large intestine are simulated in three double jacket glass reactors and the colon content is kept at $37^{\circ} \mathrm{C}$ by pumping thermostated water into the space between the glass jackets. The $\mathrm{pH}$ in the colonic units (named ascending AC, transverse TC and descending DC colon) is controlled by addition of $0.5 \mathrm{M} \mathrm{NaOH}$ and $0.5 \mathrm{M} \mathrm{HCl}$ to keep values of $5.6 \pm 0.2$ in the AC, $6.3 \pm 0.2$ in the TC and $6.8 \pm 0.2$ in the DC compartments. When the digested content of the small intestine is transferred to the proximal colon compartment (AC), the transit of colonic content between the AC, TC and DC compartments is simultaneously initiated at the same flow rate. The intestinal and colonic vessels contain ports for transit of intestinal content, sampling, continuous flushing of nitrogen and $\mathrm{pH}$ and temperature control. 
The SIMGI design is aimed to dynamically operate with the five units simulating the whole gastrointestinal process. In addition, the SIMGI software allows the work of the stomach and the small intestine in a continuous way to study food digestion and at the same time running in parallel, direct feeding of the small intestine and the transit to the colonic vessels to study microbial community development and metabolism. In this way, the system is flexible and adaptable to each need of experimental approach.

\subsubsection{Stability and Reproducibility of the System}

The SIMGI model has been recently developed and, therefore, it has a short history in evaluating digestion of food components and/or the effects of diet on modulating the gut microbiota and its metabolic activity. The fermentative module of the system (AC, TC and DC compartments) follows the concept of multiple connected, anaerobic, $\mathrm{pH}$-controlled vessels. Multi-compartment fermentation models are usually represented by three-stage culture reactors as initially designed by Gibson et al. (1988). This feature allows these models to reproduce differences from proximal (characterized by acidic $\mathrm{pH}$ and carbohydrate-excess conditions) to distal colonic regions (showing a carbohydrate-depleted and non-acidic environment). The validation of three-stage culture fermentation models to simulate the ascending, transverse and descending colon conditions have been earlier described by Macfarlane et al. (1998) and Molly et al. (1994).

The biological functioning of these fermentation models requires the development of a colon region-specific microbial community that needs to be stabilized before starting any experimental approach. This initial stage allows the microbial evolution in the three reactors from a fecal inoculum to a colon region-specific microbiota (Possemiers et al. 2004; Macfarlane et al. 1998). In addition, the stabilization period is required to provide a steady-state environment where the composition and metabolism of the microbial community can be evaluated during long-term experimental dietary interventions.

The evolution of the gut microbiota in the AC, TC and DC compartments of the SIMGI model was followed during a 2-week stabilization period (Barroso et al. 2015). Results indicated that from day 8 onwards the microbial DGGE fingerprints of samples from the same compartment clustered together and that at 14 days the microbial communities reached the steady state. The counts of some representative microbial groups analyzed by quantitative PCR (qPCR) in the AC, TC and DC compartments are shown in Fig. 28.2. Differences observed between the compartments indicated that Bacteroides were more representative in the AC and TC compartments than in the DC reactor, whereas the butyrate-producing groups C. leptum and Ruminoccocus were less represented in the proximal colon compartment (AC) than in the distal vessels (TC and DC). This specific microbiota composition during the stabilization period has also been observed in the three-stage culture model SHIME (Barroso et al. 2014; Van den Abbeele et al. 2010). 


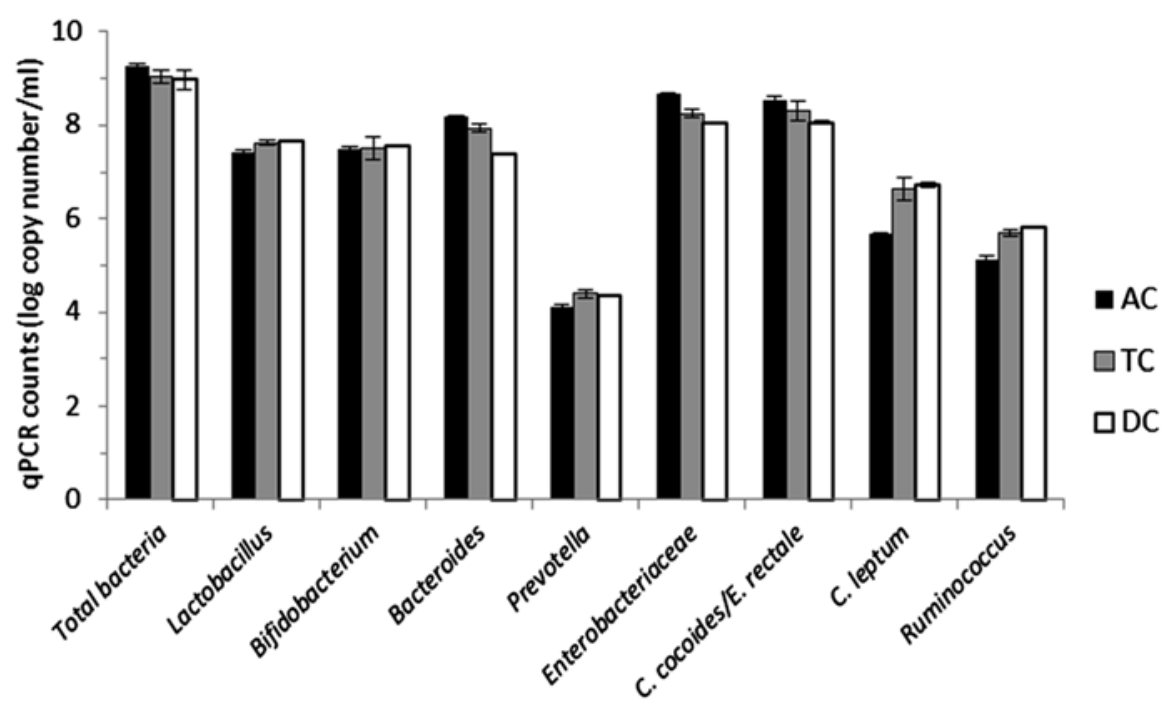

Fig. 28.2 Mean \pm SD of qPCR counts (log copy number $/ \mathrm{mL}$ ) for the microbial groups analyzed in the ascending (AC), transverse (TC) and descending colon (DC) compartments of the SIMGI after 2 weeks of stabilization

\subsubsection{Relevance to Human In Vivo Situation}

The suitability of the SIMGI fermentation model to reproduce human conditions associated to changes in dietary lifestyles has been evaluated by the simulation of an obesity-associated microbiota in this in vitro system (unpublished results). For this purpose, the three colonic reactors were inoculated with fecal microbiota from an overweight individual after which the system was feeding daily with a high energy nutritive medium for 2 weeks (microbial stabilization period). Increase of energy in the nutritive medium was achieved by increasing the content of simple sugars (fructose), simulating high consumption of sugar-sweetened beverages, and of carbohydrates from simple starches (maize and potato). After the stabilization period a dietary intervention during 7 days was performed by lowering energy of the nutritive medium in order to observe possible changes in microbial composition and metabolism induced by a sharp shift in the diet. This low energy diet was obtained by suppression of simple carbohydrates and reduction of the content of readily fermentable starches. The selection of components of the nutritive media was based on the rational design of media with different energy content described by Payne et al. (2012) to compare the impact of dietary energy on gut microbiota in a three-stage in vitro continuous fermentation model that uses fecal microbiota immobilized within a porous, non-biodegradable polysaccharide matrix (Cinquin et al. 2006). 

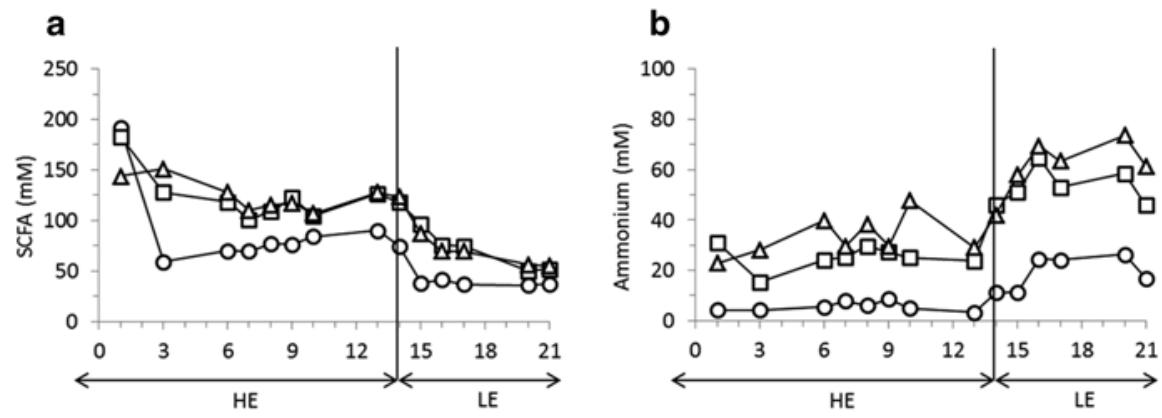

Fig. 28.3 Changes in concentration (mM) of SCFAs (a) and ammonium (b) in the ascending (AC; circles), transverse (TC; squares) and descending colon (DC; triangles) compartments of the SIMGI at different times after inoculation and feeding with high energy (HE) and low energy (LE) diets

The comparison of short chain fatty acids (SCFA) and ammonium formation under high energy (microbiota stabilization period) and low energy (dietary intervention) diets is shown in Fig. 28.3. Shift to a low energy diet resulted in a twofold decrease in the average content of total SCFA of the three colon compartments compared to the high energy intake period. Additionally, the shift from high to low energy medium caused a twofold increase in the ammonium content of the distal colon compartments (TC and DC) and a remarkable sixfold increase in the proximal colon compartment (AC). The SFCA and ammonium results could be compared with in vivo data from obese subjects, where a significant decrease of SCFA and increase of proteolytic products were observed when the individuals consumed diets high in protein and reduced in total carbohydrates (Russell et al. 2011).

\subsubsection{Quality in Relation to Other Models with the Same Applicability}

The SIMGI is designed to simulate not only the fermentation process as described above, but also the process of digestion. This is a competitive advantage compared to other systems that simulate digestion and fermentation operating in separate modules (TIM-1 and TIM-2). The whole system is computer controlled through an operator panel and programmable logic controller. It can be set up to sequentially proceed (continuously or feeding the system from 1 to 6 times daily) from the operation of food intake into the stomach throughout the delivery of distal colon content to waste. The system differs from the SHIME model in that the stomach uses peristaltic movements for mixing the ingested food with gastric fluids. Additionally, the computer software allows the definition of $\mathrm{pH}$ acidification curves that can be set up according to results obtained from in vivo studies (Marteau et al. 1990). The stomach emptying is programmable to follow the equation described by Elashoff et al. (1982) that allows the modification of the shape of the emptying curve depending 
on liquid, semisolid or solid foods. This process implies that the gastric content will be delivered to the small intestine at different $\mathrm{pH}$ values (up to $\mathrm{pH}$ 2). This is an important feature to take into account when evaluating probiotic survival growth under gastric and duodenal conditions (Fernández de Palencia et al. 2008) that would enable their eventual functionality when reaching the large intestine. Another distinctive characteristic of the SIMGI is that the small intestine and the three colon reactors are continuously flushed with nitrogen inducing anaerobic conditions for the oxygen-sensitive intestinal microbial communities, thus allowing a permanent anaerobic atmosphere.

\subsection{General Protocol}

The operation of the SIMGI as a fermentation model mainly follows the protocols established for multi-compartment culture reactors. For most of dietary interventions, the choice of the nutritive medium can be based on the medium developed by Macfarlane et al. (1998). Therefore, the three colonic vessels which will be inoculated with fecal samples are filled and pre-conditioned with the nutritive medium that will be fed to the system during the microbial stabilization period in a volume of 250, 400 and $300 \mathrm{~mL}$ for the AC, TC and DC compartments, respectively. The volumes of the colonic reactors and the transit of colonic content between compartments, three times daily at a flow rate of $5 \mathrm{~mL} / \mathrm{min}$, are intended to give an overall residence time of $76 \mathrm{~h}$, adapting the conditions already standardized in the SHIME model (De Boever et al. 2000; Molly et al. 1993; Van den Abbeele et al. 2010). Further on, the AC, TC and DC vessels are inoculated with $20 \mathrm{~mL}$ of a fresh $20 \%$ $(\mathrm{w} / \mathrm{v})$ human fecal slurry prepared in anaerobic conditions with sodium phosphate buffer (0.1 M, pH 7.0), containing $1 \mathrm{~g} / \mathrm{L}$ sodium thioglycolate as reducing agent, as described by De Boever et al. (2000). The inoculated AC, TC and DC vessels are allowed to equilibrate overnight in batch conditions at $37^{\circ} \mathrm{C}$. The $\mathrm{pH}$ in the colonic compartments is controlled by addition of $0.5 \mathrm{M} \mathrm{NaOH}$ and $0.5 \mathrm{M} \mathrm{HCl}$ to keep values of $5.6 \pm 0.2$ in the $\mathrm{AC}, 6.3 \pm 0.2$ in the TC and $6.8 \pm 0.2$ in the DC. The temperature is kept at $37^{\circ} \mathrm{C}$ by pumping water into the space between the double glass jackets of the reactor vessels. The development and stabilization of the microbial community until steady-state conditions in the three colon vessels is approached by feeding the small intestine compartment with nutritive medium $(75 \mathrm{~mL}, \mathrm{pH} 2)$ mixed with pancreatic juice $\left(40 \mathrm{~mL}\right.$ of a solution of $12 \mathrm{~g} / \mathrm{L} \mathrm{NaHCO}_{3}, 6 \mathrm{~g} / \mathrm{L}$ oxgall dehydrate fresh bile and $0.9 \mathrm{~g} / \mathrm{L}$ porcine pancreatine) three times a day during 14 days (Van den Abbeele et al. 2010). The small intestine digestion is performed during $2 \mathrm{~h}$ at $37{ }^{\circ} \mathrm{C}$ and the whole content of the vessel is automatically transferred at a flow rate of $5 \mathrm{~mL} / \mathrm{min}$ to the proximal colon compartment (AC). The transfer of intestinal content between the reactors is initiated to operate simultaneously in order to keep constant the AC, TC and DC reactor volumes. The process of stabilization 
of the colon microbiota in the three-stage reactors of the SIMGI model is usually reached after 14 days of running the system under the described conditions. This 2-week stabilization period allows the development of the fecal microbiota to colon region-specific microbial communities. After this period, the system provides a steady-state microbial environment ready to be evaluated under different experimental dietary interventions.

\subsection{Controls to Test Stability and Performance of the Model}

The SIMGI model is entirely under computer control that is conducted through an operator panel and a programmable logic controller Unitronics' Vision $120^{\mathrm{TM}}$. The software offers several operating modes: (1) the whole gastrointestinal tract; (2) independent experiments in parallel with stomach and small intestine in one module and the three-stage colon reactors in the other; and (3) the same but with the small intestine working with the colon reactors. If needed, each compartment can operate individually one by one. Instructions for setting up the working $\mathrm{pH}$ values, temperature, peristaltic or rotation frequency, volume and flow rates of fluids, etc., are all set before starting the experiments as well as the number of daily repetitions of the process. All the parameters are registered and errors (if any) are documented. For the fermentation process, the three reactors are equipped with pumps that supply $\mathrm{NaOH}$ and $\mathrm{HCl}$ to control the consigned $\mathrm{pH}$ values. The representation on line of $\mathrm{pH}$ curves and volumes of acid and base added to the compartments are indicative of microbial development and metabolism. This information is crucial as control of the process since the analyses of metabolites and fermentation products are delayed and not measured online. Occasionally, general plate counts can be performed as an additional control of microbial viability. The system, however, can be upgraded to analyze on line the formation of gas (ammonium, $\mathrm{H}_{2}, \mathrm{CO}_{2}$ and $\mathrm{CH}_{3}$ ).

\subsection{Read-Out of the System}

The system displays sampling ports between compartments to remove aliquots of colon content without disrupting the anaerobic conditions. The primary information searched during the set-up of the system and the diet interventions is the general evolution of the gut microbial community and the quantification of the targeted microbial groups and their metabolites. Analyses of colon microbiota is based on molecular biology tools (PCR-DGGE, qPCR, metagenomics, etc.), whereas general plate counts are only occasionally performed as a control of microbial viability. The choice of non-culturable microbial methods is in accordance with the large number of gut bacterial groups that are non-culturable by conventional techniques due to their generally fastidious growth requirements (Allen-Vercoe 2013). 
The stabilization of the microbial community until steady-state conditions in the three colonic reactors and its further development during dietary interventions are evaluated by sampling and measuring the production of SCFA, branched chain fatty acids (BCFA) and ammonium over time. These metabolites are considered representative of fermentative and proteolytic microbial metabolism. Depending on the conditions assayed (diet interventions, development of microbial communities representative of dysbiosis associated to intestinal pathologies, etc.), specialized analytical methodologies targeting the formation of specific intermediate and end products are currently being developed to be incorporated to the system (unpublished results).

\subsection{Advantages, Disadvantages and Limitations of the System}

The advantage of the SIMGI model is associated to its flexible-modulating characteristics and the automated control of the working parameters that can be adjusted to simulate physiological conditions. The combination of peristaltic movements and controlled emptying of the gastric and small intestine compartments are advantages of the system in comparison to other multicompartmental models. The SIMGI model has not yet incorporated devices simulating the gut microbiota-host interactions. Therefore, assays for evaluating this type of crucial interaction is currently approached by co-culturing colon-region specific microbiota suspensions from the AC, TC and/or DC vessels with epithelial or immune cells. The incubation of this SIMGI complex colonic microbiota with Caco-2 cells has shown not to disturb the epithelial barrier integrity (unpublished results). Moreover, the SIMGI microbiota has demonstrated to induce the phenotypical maturation of human monocytederived dendritic cells (unpublished results). However, a limitation of the SIMGI model is the lack of devices to evaluate the formation of microbial biofilms adhering to the colonic epithelium. The simulation of intestinal absorption to remove end products of microbial metabolism is also a limitation of the system to prevent inhibition of the colon microbiota. Both drawbacks of the system are on the way to be overcome by setting up microbial/mucosa interfaces in the SIMGI lumen and by including dialysis devices between compartments.

In summary, the fully automation of the SIMGI model allows precise control of the environmental parameters that simulate the gastrointestinal tract. This multistage dynamic model has demonstrated to reproduce complex and stable microbial communities and it can be used as a tool for studying the effects of diet or food components on modulating the gut microbiota and its metabolic activity.

Open Access This chapter is distributed under the terms of the Creative Commons Attribution Noncommercial License, which permits any noncommercial use, distribution, and reproduction in any medium, provided the original author(s) and source are credited. 


\section{References}

Allen-Vercoe E (2013) Bringing the gut microbiota into focus through microbial culture: recent progress and future perspective. Curr Opin Microbiol 16:625-629

Barroso E, Cueva C, Peláez C, Martínez-Cuesta MC, Requena T (2015) Development of human colonic microbiota in the computer-controlled dynamic SIMulator of the gastrointestinal tract SIMGI. LWT Food Sci Technol 61:283-289

Barroso E, Van de Wiele T, Jiménez-Girón A, Muñoz-González I, Martín-Alvárez PJ, MorenoArribas MV, Bartolomé B, Peláez C, Martínez-Cuesta MC, Requena T (2014) Lactobacillus plantarum IFPL935 impacts colonic metabolism in a simulator of the human gut microbiota during feeding with red wine polyphenols. Appl Microbiol Biotechnol 98:6805-6815

Cinquin C, Le Blay G, Fliss I, Lacroix C (2006) Comparative effects of exopolysaccharides from lactic acid bacteria and fructooligosaccharides on infant gut microbiota tested in an in vitro colonic model with immobilized cells. FEMS Microbiol Ecol 57:226-238

De Boever P, Deplancke B, Verstraete W (2000) Fermentation by gut microbiota cultured in a simulator of the human intestinal microbial ecosystem is improved by supplementing a soygerm powder. J Nutr 130:2599-2606

Elashoff JD, Reedy TJ, Meyer JH (1982) Analysis of gastric-emptying data. Gastroenterology 83:1306-1312

Fernández de Palencia P, López P, Corbí AL, Peláez C, Requena T (2008) Probiotic strains: survival under simulated gastrointestinal conditions, in vitro adhesion to Caco-2 cells and effect on cytokine secretion. Eur Food Res Technol 227:1475-1484

Gibson GR, Cummings JH, Macfarlane GT (1988) Use of a three-stage continuous culture system to study the effect of mucin on dissimilatory sulfate reduction and methanogenesis by mixed populations of human gut bacteria. Appl Environ Microbiol 54:2750-2755

Macfarlane GT, Macfarlane S, Gibson GR (1998) Validation of a three-stage compound continuous culture system for investigating the effect of retention time on the ecology and metabolism of bacteria in the human colon. Microb Ecol 35:180-187

Marteau P, Flourié B, Pochart P, Chastang C, Desjeux JF, Rambaud JC (1990) Effect of the microbial lactase (EC 3.2.1.23) activity in yoghurt on the intestinal absorption of lactose: an in vivo study in lactase-deficient humans. Br J Nutr 64:71-79

Molly K, Van de Woestyne M, Verstraete W (1993) Development of a 5-step multichamber reactor as a simulation of the human intestinal microbial ecosystem. Appl Microbiol Biotechnol 39:254-258

Molly K, Van de Woestyne M, De Smet I, Verstraete W (1994) Validation of the simulator of the human intestinal microbial ecosystem (SHIME) reactor using microorganism-associated activities. Microb Ecol Health Dis 7:191-200

Payne AN, Chassard C, Banz Y, Lacroix C (2012) The composition and metabolic activity of child gut microbiota demonstrate differential adaptation to varied nutrient loads in an in vitro model of colonic fermentation. FEMS Microbiol Ecol 80:608-623

Possemiers S, Verthé K, Uyttendaele S, Verstraete W (2004) PCR-DGGE-based quantification of stability of the microbial community in a simulator of the human intestinal microbial ecosystem. FEMS Microbiol Ecol 49:495-507

Russell WR, Gratz SW, Duncan SH, Holtrop G, Ince J, Scobbie L, Duncan G, Johnstone AM, Lobley GE, Wallace RJ, Duthie GG, Flint HJ (2011) High-protein, reduced-carbohydrate weight-loss diets promote metabolite profiles likely to be detrimental to colonic health. Am J Clin Nutr 93:1062-1072

Van den Abbeele P, Grootaert C, Marzorati M, Possemiers S, Verstraete W, Gérard P, Rabot S, Bruneau A, El Aidy S, Derrien M, Zoetendal E, Kleerebezem M, Smidt H, Van de Wiele T (2010) Microbial community development in a dynamic gut model is reproducible, colon region specific, and selective for Bacteroidetes and Clostridium cluster IX. Appl Environ Microbiol 76: $5237-5246$ 